\title{
Long non-coding RNA TUG1 promotes the proliferation of colorectal cancer cells through regulating Wnt/ $\beta$-catenin pathway
}

\author{
CHUN HONG XIAO ${ }^{1}$, HAI ZHONG YU ${ }^{2}$, CHUN YAN GUO ${ }^{2}$, ZHI MEI WU ${ }^{2}$, \\ HONG YAN CAO ${ }^{2}$, WEI BING $\mathrm{LI}^{2}$ and JIAN FEN YUAN ${ }^{2}$ \\ ${ }^{1}$ Department of Clinical Laboratory, Nantong Cancer Hospital; ${ }^{2}$ Department of Clinical Laboratory, \\ Traditional Chinese Medicine Hospital, Nantong, Jiangsu 226001, P.R. China
}

Received November 10, 2016; Accepted January 8, 2018

DOI: $10.3892 / \mathrm{ol} .2018 .9259$

\begin{abstract}
The long non-coding RNA taurine up-regulated gene 1 (TUG1) has been shown to be dysregulated in various types of malignant cancer; however, its underlying mechanism of action has not been fully elucidated. The present study aimed to investigate the biological role and clinical significance of TUG1 in the progression of colorectal cancer (CRC). A reverse transcription-quantitative polymerase chain reaction assay was used to evaluate TUG1 expression in tissues from patients with CRC. The effect of TUG1 on cell viability of CRC cells using MTT assay. The influence of TUG1 on tumorigenesis was monitored using an in vivo xenograft model. The status of the $\mathrm{Wnt} / \beta$-catenin signaling pathway was evaluated using immunofluorescence, western blotting and luciferase reporter assays. The results demonstrated that the expression of TUG1 was positively associated with the pathological grade and clinical stage of CRC patients. Knockdown of TUG1 inhibited the proliferation of CRC cells and attenuated the activity of $\mathrm{Wnt} / \beta$-catenin pathway in CRC cells. In addition, TUG1 knockdown inhibited the tumorigenicity in the in vivo CRC xenograft model, as well as the nuclear localization of $\beta$-catenin and downstream gene transcription. Taken together, the data of the present study highlighted the pivotal role of the TUG1-Wnt/ $\beta$-catenin signaling pathway in CRC, which could be targeted to improve the therapeutic efficacy of CRC.
\end{abstract}

\section{Introduction}

Colorectal cancer (CRC) is the third most common malignancy in the world (1). Numerous causes lead to the occurrence of $\mathrm{CRC}$, including the mutation or alteration of expression of multiple genes, including proto-oncogenes and

Correspondence to: Dr Jian Fen Yuan, Department of Clinical Laboratory, Traditional Chinese Medicine Hospital, 41 JianShe Road, Nantong, Jiangsu 226001, P.R. China

E-mail: sci153@sina.com

Key words: long non-coding RNA, taurine up-regulated gene 1, colorectal cancer, proliferation, Wnt/ $\beta$-catenin signaling pathway tumor suppressor genes. Whether the oncogenes controlling cell proliferation are highly expressed or the tumor suppressor genes are deleted or mutated, it can lead to the occurrence and development of CRC (2). However, the underlying molecular mechanism causing tumor initiation in $\mathrm{CRC}$ remains elusive.

Long non-coding RNAs (lncRNAs) are a class of non-protein coding transcripts $>200$ nucleotides in length (3). Evidence indicates that IncRNAs play critical roles in the cancer development by regulating the proliferation, metastasis, drug resistance, and apoptosis of cancer cells (4-7). The lncRNA taurine up-regulated gene 1 (TUG1) was originally identified as a transcript that is upregulated by taurine, which is highly conserved in mammals and was originally detected in a genomic screen in taurine-treated mouse retinal cells (8). Evidence has revealed that TUG1 is dysregulated in several cancer types (9), including bladder carcinoma (10-12), colon cancer $(13,14)$, lung cancer (15), osteosarcoma (16), B-cell neoplasms (17) and esophageal squamous cell carcinoma (18). One recent study has demonstrated that, double-negative feedback loop between TUG1 and microRNA-145 (miR-145) promotes epithelial-to-mesenchymal transition and radioresistance in human bladder cancer cells (12). The present study aimed to investigate the biological role and clinical significance of TUG1 in the progression of CRC, providing targets for improving the therapeutic efficacy of CRC.

\section{Materials and methods}

Patient selection. The present study was a case-control pilot study developed at Nantong Hospital of Traditional Chinese Medicine (Nantong, China) with the analysis of 90 patients with CRC ( 38 female and 52 male cases; median age, 62 years old; age range, 48 to 79 years old), and 30 healthy donors (13 female and 17 male cases; median age, 61 years old; age range, 45 to 72 years old) were used as the control. Of the specimens, 63 were primary tumor (stage I and II) and 27 advanced tumor (stage III and IV) CRC. The protocols for tumor sample collection were approved by the Institutional Ethics Committee at Nantong Hospital of Traditional Chinese Medicine. Each patient provided written informed consent for inclusion in the present study. 
Cell culture and lentivirus infection. SW620 and LoVo cell lines were purchased from the American Type Culture Collection (Manassas, VA, USA). SW620 cell lines were cultured in L-15 medium (Gibco; Thermo Fisher Scientific, Inc., Waltham, MA, USA) and LoVo cell lines were cultured in F12K medium (Gibco; Thermo Fisher Scientific, Inc.). All mediums were supplemented with $10 \%$ fetal bovine serum (FBS; Gibco; Thermo Fisher Scientific, Inc.), $100 \mathrm{U} / \mathrm{ml}$ penicillin (Gibco; Thermo Fisher Scientific, Inc.) and $100 \mu \mathrm{g} / \mathrm{ml}$ streptomycin (Gibco; Thermo Fisher Scientific, Inc.). All cells were cultured at $37^{\circ} \mathrm{C}$ in a humidified atmosphere containing $5 \% \mathrm{CO}_{2}$. For the knockdown of TUG1, lentiviral vectors $(2 \mathrm{E}+8 \mathrm{TU} / \mathrm{ml})$ harboring TUG1 short hairpin RNAs (shRNAs) were constructed (Shanghai GeneChem Co., Ltd., Shanghai, China). The sequences were as follow: shRNA1, 5-GTCTGCATTGAGGATATAG-3; shRNA2, 5-GCCAAA TAACTGAAGCTAT-3 and shRNA3, 5-GTACGTGTCTTG GAAGTCT-3. The negative targeted shRNA was a scrambled sequence: 5-CCTCTAGGTAAGCATAATTTT-3. Lentiviral particles were produced by co-transfecting the expression vector Lenti-KD-shRNA/TUG1 or Lenti-KD-shRNA/NT (Shanghai GeneChem Co., Ltd., Shanghai, China) with viral particle packaging helper vector into $293 \mathrm{~T}$ cells using Lipofectamine 3000 (Thermo Fisher Scientific, Inc.). Human 293T cells (American Type Culture Collection) were cultured in Dulbecco's modified Eagle's medium supplemented with $10 \% \mathrm{FBS}, 100 \mathrm{U} / \mathrm{ml}$ penicillin and $100 \mathrm{~g} / \mathrm{ml}$ streptomycin at $37^{\circ} \mathrm{C}$ in a humidified atmosphere containing $5 \% \mathrm{CO}_{2}$. The titers of the viral particles containing Lenti-KD-shRNA/TUG1 and Lenti-KD-shRNA/NT were determined by performing 10 -fold serial dilutions in Eppendorf tubes for 8 consecutive dilutions. The dilution method was as follows: A total of $8 \mathrm{EP}$ tubes $(1.5 \mathrm{ml})$ were prepared for each lentivirus, and $90 \mu 1 \mathrm{~L}-15$ medium was added to each tube. A total of $10 \mu 1$ lentivirus stock solution was added to the first tube, mixed, and of this solution $10 \mu \mathrm{l}$ was then pipetted into the second tube and mixed again, until a final dilution of $10 \times 10^{-6}$. SW620 and LoVo cells were infected with the aforementioned packaged lentivirus. After $72 \mathrm{~h}$, the efficiencies of knockdown were determined by reverse transcription-quantitative polymerase chain reaction (RT-qPCR).

$R T$ - $q P C R$. Total RNAs were extracted from cells using RNAiso Plus reagent (Takara Biotechnology Co., Ltd., Dalian, China). The cDNA was synthesized using PrimeScript ${ }^{\mathrm{TM}}$ RT reagent kit with gDNA Eraser kit (Takara Biotechnology Co., Ltd.). The mRNA level was determined using SYBR ${ }^{\circledast}$ Premix Ex Taq ${ }^{\mathrm{TM}}$ II (Takara Biotechnology Co., Ltd.) by ABI 7500 real-time PCR system (Applied Biosystems; Thermo Fisher Scientific, Inc.). GAPDH was used as an internal control. The thermocycling conditions used were as follows: $95^{\circ} \mathrm{C}$ for $5 \mathrm{~min}$, followed by 40 two-step cycles $\left(95^{\circ} \mathrm{C}\right.$ for $20 \mathrm{sec}$ and $60^{\circ} \mathrm{C}$ for $\left.30 \mathrm{sec}\right)$. Relative mRNA levels were calculated using the $2^{-\Delta \Delta C q}$ method (19). The sequences of primers are as follows: TUG1 forward, TAGCAGTTCCCCAATCCTTG and reverse, CAC AAATTCCCATCATTCC; and GAPDH forward, CCACCC ATGGCAAATTCCATGGCA and reverse, TCTAGACGG CAGGTCAGGTCCACC.
Cell proliferation and survival assays. Cell proliferation was determined using an MTT assay kit (cat no. C0009; Beyotime Institute of Biotechnology, Haimen, China) according to manufacturer's instructions. Once the cells were cultured for $24,48,72$ and $96 \mathrm{~h}$, MTT solution ( $0.5 \%$, dissolved in dimethyl sulfoxide) was added into each well and the cells were incubated for another $4 \mathrm{~h}$ at $37^{\circ} \mathrm{C}$, followed by absorbance detection. The absorbance in each well was measured using a microplate reader at a wavelength of $490 \mathrm{~nm}$ (Bio-Rad Laboratories, Inc., Hercules, CA, USA).

Tumorigenesis assay. The animal experiments were performed in accordance with the guidelines by the U.S. National Institute of Health Guide for the Care and Use of Laboratory Animals (20). The protocol was approved by the Animal Care and Ethics Committee of Nantong Hospital of Traditional Chinese Medicine. Scramble shRNA, or TUG1 shRNA-infected SW620 or LoVo cells $\left(\sim 5 \times 10^{6}\right)$ were injected subcutaneously in the female BALB/c nude mice (4-6 weeks old, $20 \mathrm{~g}$ ) obtained from Shanghai SLAC Laboratory Animal Co., Ltd. (Shanghai, China) and maintained in pathogen-free conditions of temperature $\left(22 \pm 2^{\circ} \mathrm{C}\right)$, humidity $(55 \pm 5 \%)$ and a $12 \mathrm{~h} / 12 \mathrm{~h}$ light-dark cycle with free access to food and water. The length (L) and width (W) of each tumor was measured with calipers and the volume was calculated using the equation: $\mathrm{V}=\left(\mathrm{L} \mathrm{x} \mathrm{W}^{2}\right) \times 0.5$ for 42 days after the injection of cancer cells. Mice were sacrificed after 42 days using cervical dislocation following $\mathrm{CO}_{2}$ inhalation.

Immunofluorescence and immunohistochemical staining. The subcutaneous transplantation tumor tissues or cells (SW620 and LoVo) were fixed with 4\% paraformaldehyde for $20 \mathrm{~min}$ at room temperature, permeabilized by $0.1 \%$ Triton X-100 and washed with PBS three times followed by blocking with $10 \%$ normal goat serum plus $1 \%$ bovine serum albumin for $45 \mathrm{~min}$ at room temperature and incubated with rabbit anti- $\beta$-catenin (1:100; cat no. \#9562; Cell Signaling Technology Inc., Danvers, MA, USA) at $4^{\circ} \mathrm{C}$ overnight. After washing three times with PBS, the tissue slices were further incubated with Cy3-conjugated goat anti-rabbit (cat no. A0516; Beyotime Institute of Biotechnology) or HRP-conjugated goat anti-rabbit secondary antibodies (cat no. A0208; Beyotime Institute of Biotechnology) for $1 \mathrm{~h}$ at $37^{\circ} \mathrm{C}$. Subsequently, the slides were cover slipped with mounting medium (Dako; Agilent Technologies, Inc., Santa Clara, CA, USA) containing DAPI to counterstain the nuclei and imaged with a fluorescence or bright field microscope (at a magnification of $\mathrm{x} 100$ for immunofluorescence or $\mathrm{x} 200$ for immunohistochemical staining).

T-cell factor/lymphoid enhancer factor (TCF/LEF) promoter activity analysis. To test the LEF/TCF promoter activity, SW620 or LoVo cells were co-transfected with the recombinant plasmid pGL3-basic-LEF/TCF promoter with a control positive plasmid pRL-SV40, and the TCF/LEF promoter activity analysis was performed according to the protocol of Ji et al (21). The promoter activity was analyzed using a commercial dual-luciferase assay kit (Beyotime Institute of Biotechnology, China) according to the manufacturer's protocol. 
A

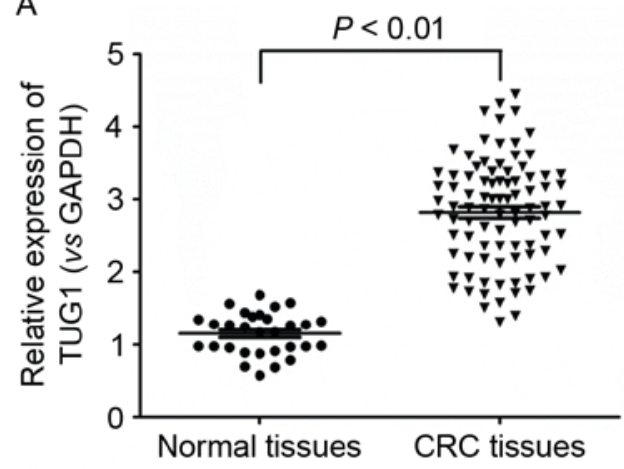

C

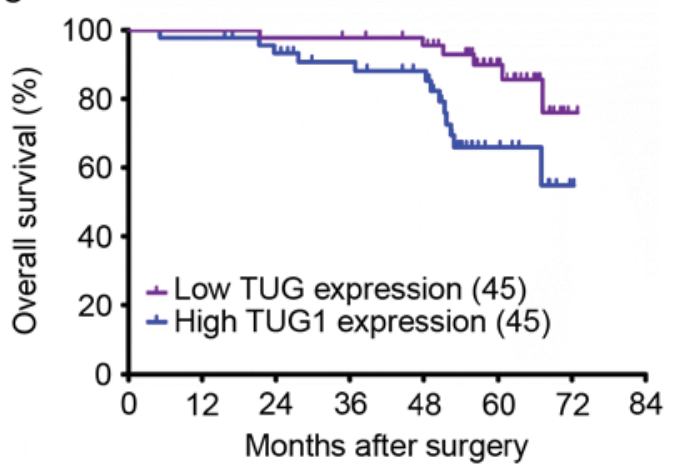

B

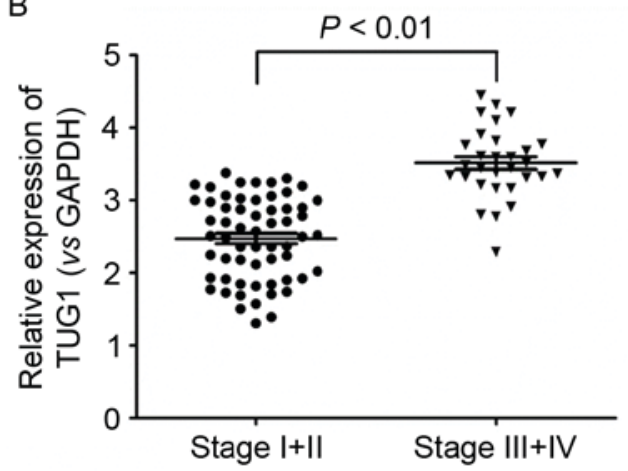

D

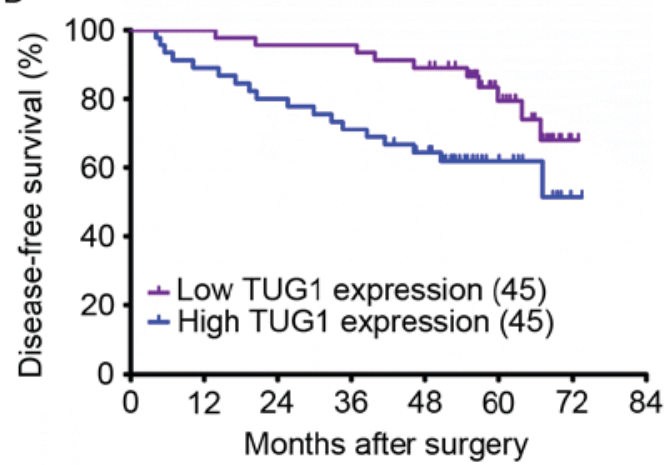

Figure 1. TUG1 expression is associated with the progression of CRC. (A) Reverse transcription-quantitative polymerase chain reaction analysis of TUG1 expression in CRC and normal tissue. (B) Analysis of TUG1 expression in patients with different stages of CRC. (C and D) Kaplan-Meier analysis of the association between TUG1 expression level and overall survival or disease-free survival rate of 90 patients with CRC, with the median expression level used as the cutoff. Patients with TUG1 expression values below the 50th percentile were classified as having lower TUG1 levels; patients with miR-195 expression values above the 50th percentile were classified as having higher TUG1 levels. ${ }^{*} \mathrm{P}<0.05$, was considered as statistically significant. TUG1, taurine up-regulated gene 1; CRC, colorectal cancer.

Western blot analysis. The protein expression levels of $\beta$-catenin were analyzed with western blot. Following transfection, cells were harvested in Radioimmunoprecipitation Assay lysis buffer (Beyotime Institute of Biotechnology, Shanghai, China) supplemented with protease inhibitor cocktail (Sigma-Aldrich; Merck KGaA, Darmstadt, Germany). A total of $40 \mu \mathrm{g}$ protein from each sample concentration determined using the BCA protein assay reagent kit (cat no. 23225; Pierce; Thermo Fisher Scientific, Inc.) and molecular weight determined by the Prestained Protein Molecular Weight Marker (cat no. 26612; Fermentas; Thermo Fisher Scientific, Inc., Pittsburgh, PA, USA, $\beta$-catenin: 92kD, $\beta$-actin: $42 \mathrm{kD}$ ] was resolved in $10 \%$ SDS-PAGE and transferred to a polyvinylidene difluoride membrane. Membranes were blocked with 5\% non-fat dry milk in TBS- $0.05 \%$ Tween-20 (TBST) buffer for $2 \mathrm{~h}$ at room temperature and incubated with primary antibodies rabbit anti- $\beta$-catenin (cat no. \#9562; Cell Signaling Technology, Inc.) and rabbit anti- $\beta$-actin (cat no. \#4970; Cell Signaling Technology, Inc.), each diluted to $1: 1,000]$ at $4^{\circ} \mathrm{C}$ overnight. After washing 3 times with TBST buffer, the membranes were incubated HRP-conjugated goat anti-rabbit secondary antibody (1:5,000; cat no. A0516; Beyotime Institute of Biotechnology) for $1 \mathrm{~h}$ at room temperature. Next, the membranes were washed, developed using Millipore Western Blot chemiluminescence HRP substrate (cat no. WBKLS0010; Merck KGaA), and images were captured a using DMI3000B inverted microscope (Leica Microsystems $\mathrm{GmbH}$, Wetzlar, Germany).
Statistical analysis. Data are presented as mean \pm standard error of the mean of at least three independent experiments. Significance of the mean between two groups was determined using an unpaired Student's t-test. Differences at different time points between groups were evaluated using a one-way analysis of variance followed by a Dunnett's test. $\mathrm{P}<0.05$ was considered to indicate a statistically significant difference. Analyses were performed using SPSS 20.0 software (IBM Corp., Armonk, NY, USA).

\section{Results}

Association between the TUG1 expression and clinicopathological characteristics in CRC. Significant upregulation of TUG1 expression was observed in CRC tissues compared with normal controls (Fig. 1A). Expression of TUG1 expression was significantly higher in stages III/IV than in stage I/II of CRC, indicating a positive association between the advanced stage of CRC and TUG1 expression (Fig. 1B). Correspondingly, the overall survival (OS) and disease-free survival (DFS) times were low in CRC patients with high TUG1 expression levels when compared with those expressing low levels of TUG1 (Fig. 1C and D). These results indicated that TUG1 might contribute to CRC development.

TUG1 increased the proliferation ability of CRC cells. To study the contribution of TUG1 to the proliferation of CRC, 
A

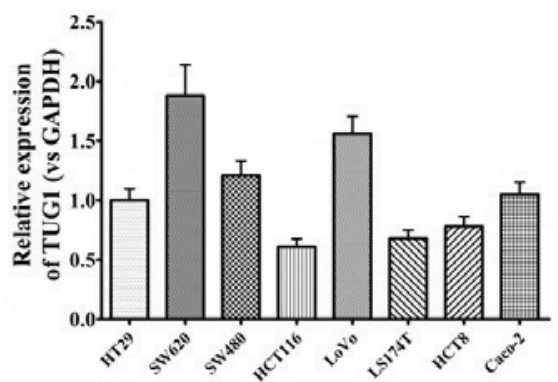

C

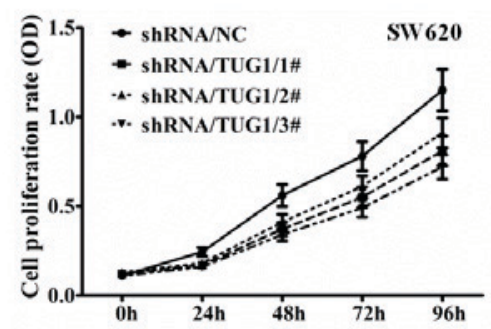

B

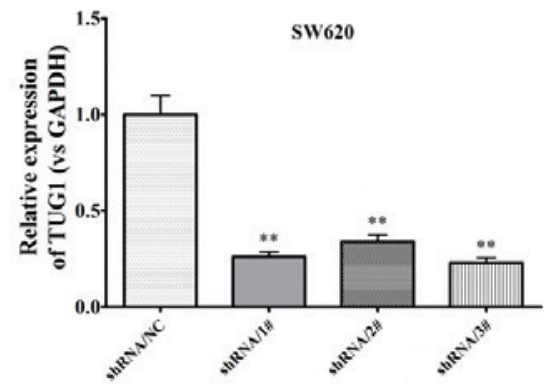

D

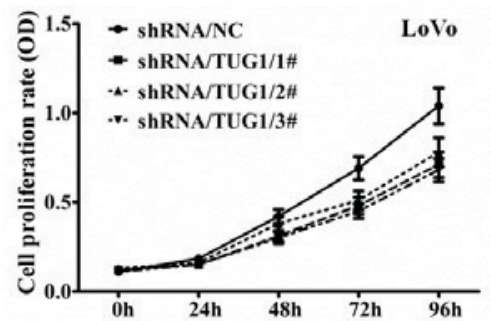

Figure 2. TUG1 increased the proliferation ability of CRC cells. (A) Reverse transcription-quantitative polymerase chain reaction analysis of TUG1 in different CRC cell lines. (B) Efficiency of different TUG1 shRNAs on the expression of TUG1. (C and D) Knockdown of TUG1 decreases the proliferation of CRC SW620 and LoVo cells. ${ }^{* *} \mathrm{P}<0.01$. TUG1, taurine up-regulated gene 1; CRC, colorectal cancer; shRNA, short hairpin RNA; OD, optical density; NC, negative control.

the expression of TUG1 was examined in the SW620 and LoVo cell lines. As depicted in Fig. 2A, the expression level of TUG1 was significant high in SW620 and LoVo cells in compared with other CRC cells. To assess the contribution of TUG1 to cellular proliferation, TUG1 expression levels were successfully knocked down in SW620 and LoVo cells (Fig. 2B), and it was found that the proliferation rate was significantly decreased in the two cell lines (Fig. 2C and D). These data indicated that TUG1 expression contributes to the proliferation of CRC cells.

TUG1 enhanced the activation of the Wnt/ $\beta$-catenin signaling pathway. The $\mathrm{Wnt} / \beta$-catenin signaling pathway is involved in the promotion of proliferation of CRC cells. To investigate the mechanism underlying the regulation by TUG1, the expression and location of $\beta$-catenin was examined in CRC cells treated with TUG1 shRNA by immunofluorescence staining. As shown in Fig. 3A and B, TUG1 shRNA significantly inhibited the expression of $\beta$-catenin protein in the nucleus of SW620 and LoVo cells. Furthermore, the western blot analysis also revealed that the levels of $\beta$-catenin were downregulated in the nuclear compartment when the CRC cells were treated with TUG1 shRNA (Fig. 3C and D). However, transfection with TUG1 shRNA did not affect the total expression of $\beta$-catenin (Fig. 3C and D). Accordingly, the TCF/LEF reporter assay also demonstrated that the Wnt/ $\beta$-catenin signaling was significantly inhibited by TUG1 shRNA (Fig. 3E and F).

TUG1 promoted the tumorigenicity of CRC cells. To confirm the aforementioned effects of TUG1 on CRC cells in vivo, the effect of knockdown of TUG1 on the tumorigenicity of SW620 and LoVo cells was analyzed using the subcutaneous xenograft mouse model. As depicted in Fig. 4A and B, the size of tumors was greatly reduced at each time point in mice injected with
CRC cells transfected with TUG1 shRNA. At day 42, the tumors were taken out and measured. The size and weight of tumors were significantly decreased by knockdown of TUG1 in SW620 and LoVo cells (Fig. 4C and D). These results indicated that TUG1 could promote the tumorigenicity of CRC cells.

TUG1 promoted the nuclear localization of $\beta$-catenin in patient tissues and the xenograft model. Since TUG1 could regulate the nuclear localization of $\beta$-catenin in CRC cells, $\beta$-catenin expression was examined in different stages of CRC patients. Consistent with the results of the aforementioned in vitro experiments, immunohistochemical staining revealed that $\beta$-catenin expression was higher in the nuclei of patients with stage III and IV CRC tumor tissues than that in stage I and II CRC patients (Fig. 5A and B).

Immunohistochemical staining and western blot analysis revealed that there was lower $\beta$-catenin expression in the nuclei from the excised tumors in mice generated by CRC cells transfected with TUG1 shRNA (Fig. 5C and D). These data indicated that TUG1 promotes the nuclear translocation of $\beta$-catenin in advanced CRC patients (stage III and IV) and in the in vivo mice model.

\section{Discussion}

CRC, as one of the most common malignant tumors in the world, is typically classified as cancer of the proximal colon, distal colon or rectum (1). A considerable number of patients with CRC are diagnosed at advanced stages and have a poor prognosis (1). The treatment modalities for CRC include surgical resection, chemotherapy, immunotherapy and other alternative methods such as traditional Chinese medicine (22). Numerous causes can bring about the occurrence of $\mathrm{CRC}$, including the mutation or altered expression of proto-oncogenes and tumor 
A

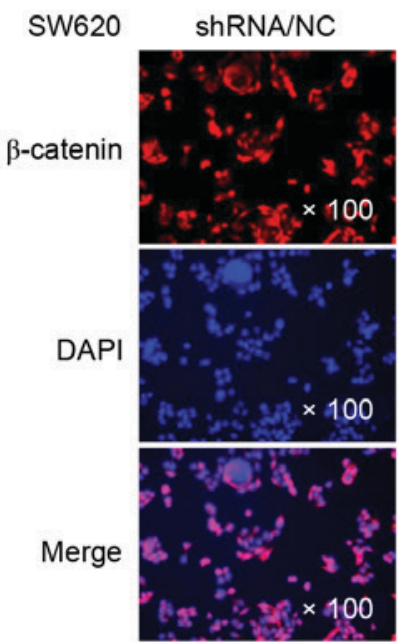

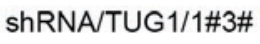
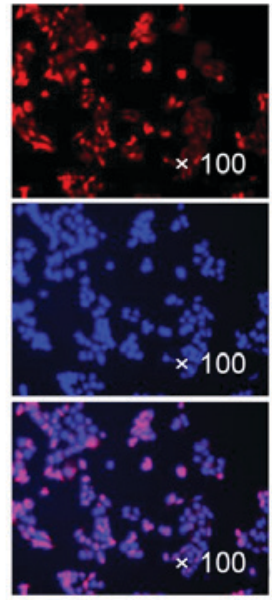

C SW620

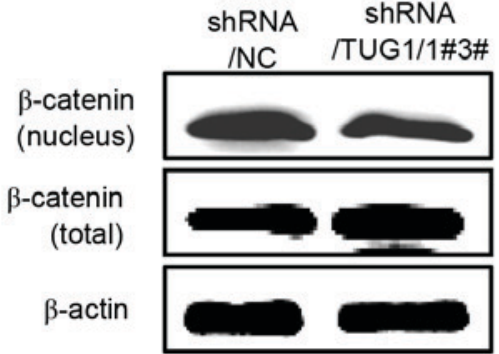

E

SW620

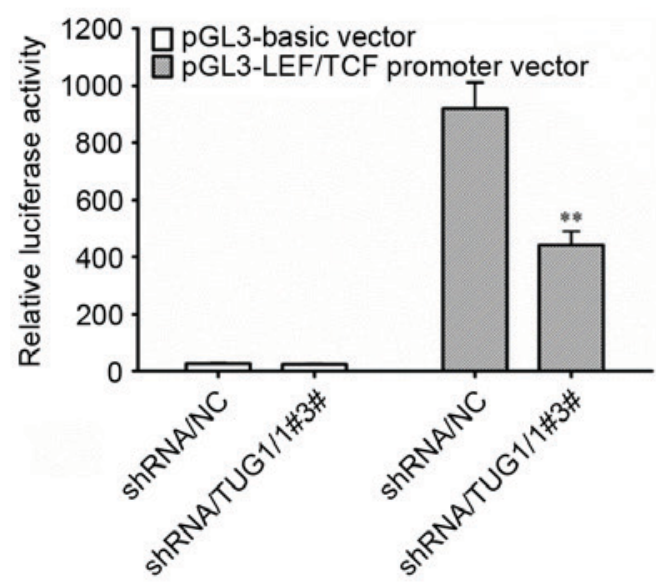

B

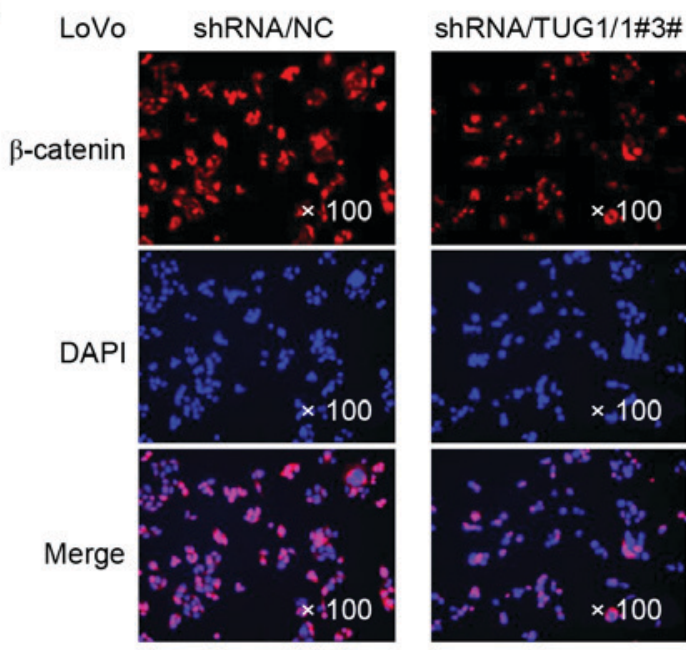

D

LoVo

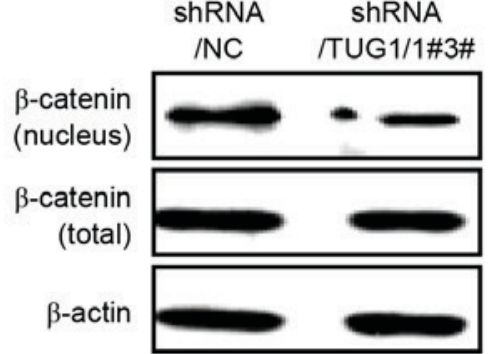

F

LoVo

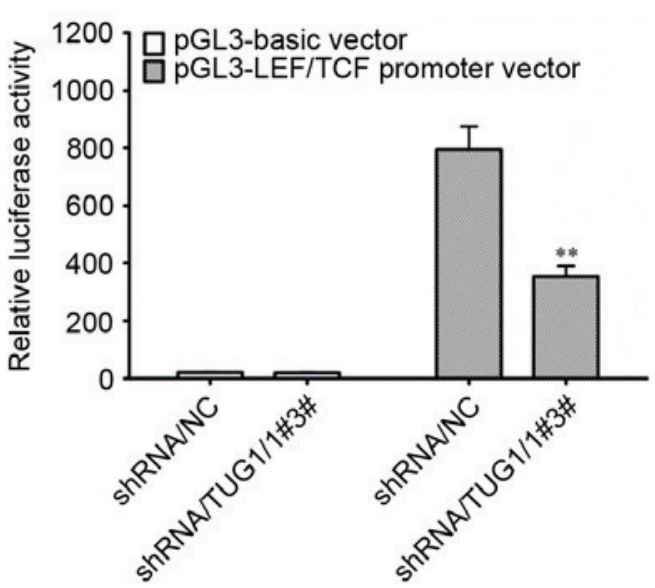

Figure 3. TUG1 enhanced the activation of Wnt/ $\beta$-catenin signaling pathway. (A and B) Representative images showing the nuclear localization of $\beta$-catenin in SW620 and LoVo cells transfected with TUG1 shRNA or scramble shRNA. The magnification level is indicated on the figure. (C and D) Western blot analysis of the $\beta$-catenin levels in the nuclear fraction from CRC cells transfected with TUG1 shRNA or scramble shRNA. $\beta$-actin was the internal reference. (E and F) TCF/LEF reporter assay for detecting the activation of Wnt/ $\beta$-catenin signaling in SW620 and LoVo cells transfected with TUG1 or NC scramble shRNA. ${ }^{* *} \mathrm{P}<0.01$. TUG1, taurine up-regulated gene 1; CRC, colorectal cancer; shRNA, short hairpin RNA; NC, negative control; TCF/LEF, T-cell factor/lymphoid enhancer factor.

suppressor genes. However, to the best of our knowledge, the underlying molecular mechanism leading to tumor initiation in CRC remains unclear.

Evidence has demonstrated that lncRNAs serve notable roles in different aspects of cancer biology and can contribute to the development of different cancer types (9). The lncRNA TUG1 was originally characterized as a transcript whose expression was upregulated by taurine, and was later observed to be overexpressed in a number of cancer tissues, including bladder carcinoma (10-12), colon cancer $(13,14)$, lung cancer $(15)$, osteosarcoma (16), B-cell neoplasms (17), and esophageal squamous cell carcinoma (18). Tan et al (12) demonstrated that
TUG1 promoted bladder cancer cell metastasis and radioresistance via negatively regulating miR-145 expression by acting as a miRNA sponge. In esophageal carcinoma, TUG1 promoted proliferation and migration in vitro (18-22). Gezer et al (23), found that TUG1 were highly enriched in secreted exosomes of HeLa and MCF-7 cells. These data indicated that TUG1 might promote cancer progression. Nevertheless, the function and mechanism of TUG1 in CRC remain unknown. The present study demonstrated that the expression of TUG1 was upregulated in the tumor tissues of advanced-stage patients with CRC, and was positively associated with the mortality of CRC patients. In addition, high TUG1 expression was also 
A

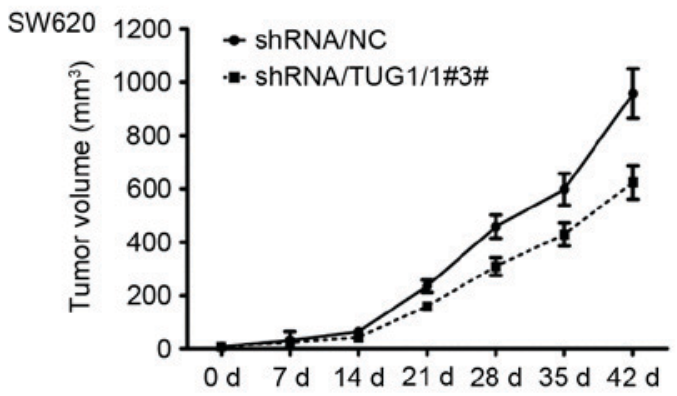

C

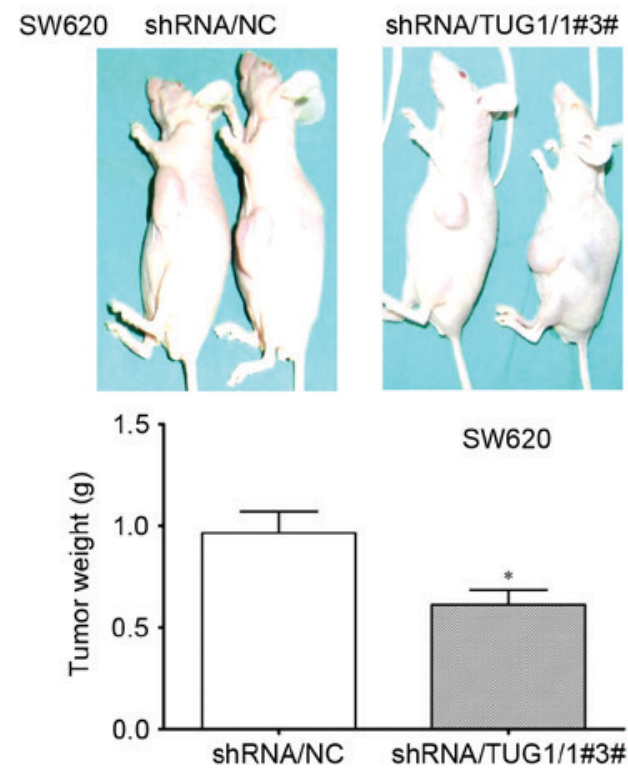

B

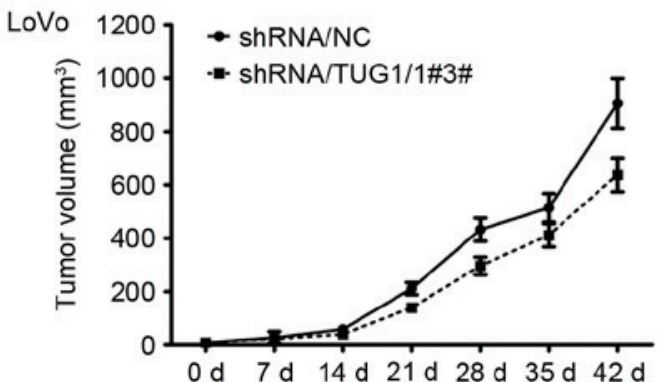

D
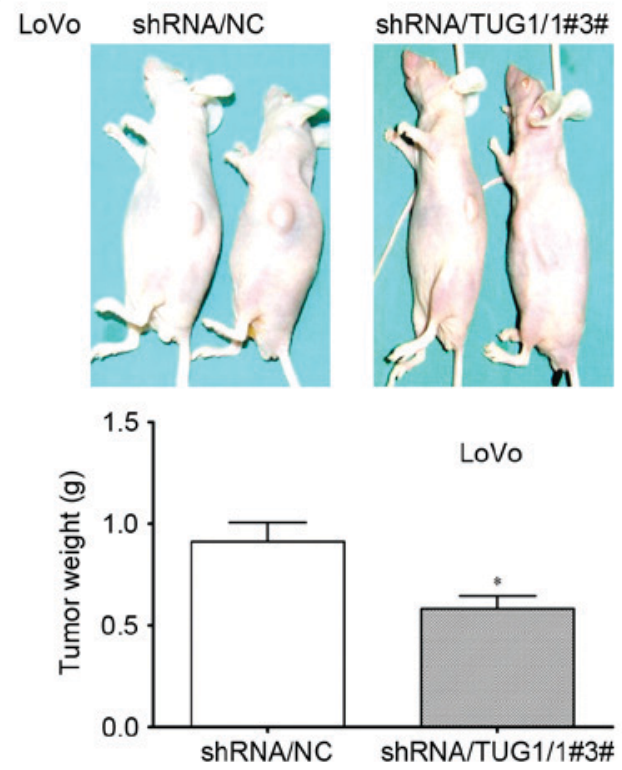

Figure 4. TUG1 promoted the tumorigenicity of CRC cells. (A and B) SW620 and LoVo cells transfected with NC scramble shRNA or shRNA TUG1 were injected subcutaneously into nude mice $(n=8)$. The length and width of the tumors were measured every 7 days. (C and $D)$ After 42 days, mice were killed for determination of tumor weights. "P<0.05. TUG1, taurine up-regulated gene 1; CRC, colorectal cancer; shRNA, short hairpin RNA; NC, negative control.

A

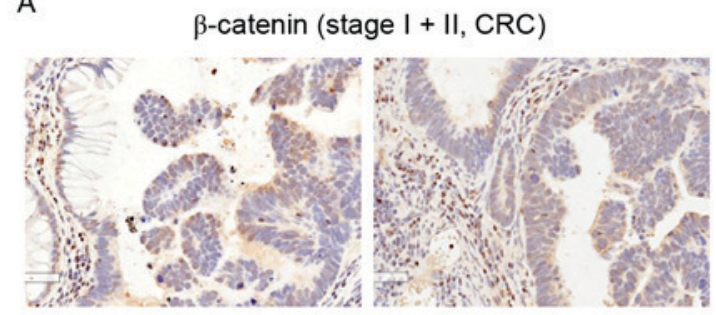

C

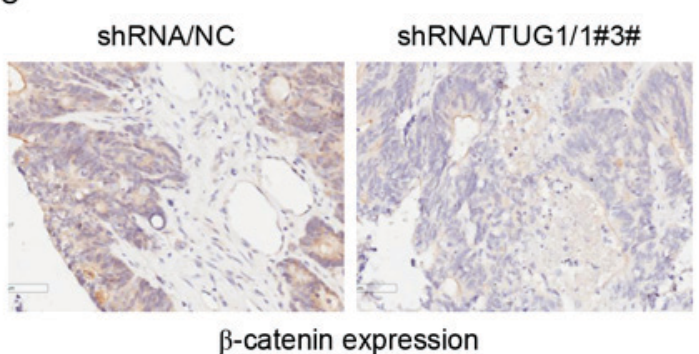

B

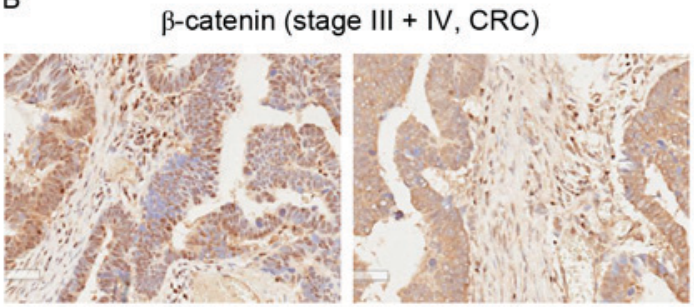

D

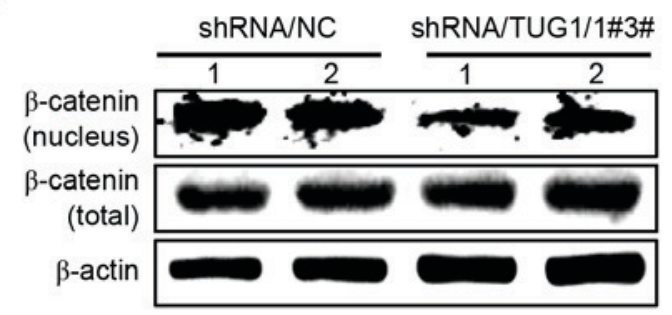

Figure 5. TUG1 promoted the nuclear localization of $\beta$-catenin in the xenograft model. (A and B) Immunohistochemical staining of tumor tissues from different stages of CRC patients using an anti- $\beta$-catenin antibody. (C) Immunohistochemical staining of tumor tissues from the excised tumors generated by CRC cells transfected with scramble shRNA or TUG1 shRNA. (D) Western blot analysis of the $\beta$-catenin levels in the two tumor tissues from the excised tumors generated by CRC cells transfected with scramble shRNA or TUG1 shRNA. TUG1, taurine up-regulated gene 1; CRC, colorectal cancer; shRNA, short hairpin RNA; NC, negative control. 
detected in several CRC cells, including SW620 and LoVo cells. Together, these data indicated that TUG1 might serve notable roles in the development of CRC, although the detailed mechanism was unclear.

$\beta$-catenin, as the key transcriptional activation factor of the Wnt/ $\beta$-catenin signal pathway, can, when activated, translocate to the nucleus from the cytoplasm in coordination with other transcription factors, including TCF, LEF, Pygo, and B-cell CLL/lymphoma 9 to activate its target genes, including cyclin D1, c-Myc, cluster of differentiation 44, and matrix metalloproteinase-7 (MMP-7), which serve notable roles in the initiation and development of tumors (24-27). The current study demonstrated that the shRNA-mediated knockdown of TUG1 evidently reduced the proliferation abilities of CRC cells. Knockdown of TUG1 inhibited the translocation of $\beta$-catenin from the cytoplasm to the nucleus, resulting in decreases in the expression of c-Myc and MMP-7, whereas overexpression of TUG1 brought about the contrary. The TCF/LEF family is a group of transcription factors that is involved in the Wnt/ $\beta$-catenin signaling pathway, which bind to DNA through a high-mobility group domain and recruit the co-activator $\beta$-catenin to enhancer elements of target genes (28). By using $\mathrm{LEF} / \mathrm{TCF}$ promoter dual-luciferase reporter construct, it was demonstrated that knockdown of TUG1 downregulated the promoter activity of LEF/TCF, whereas it was enhanced by overexpression of TUG1. Together, these results indicated that, TUG1 might promote the proliferation of CRC cells by regulating the $\mathrm{Wnt} / \beta$-catenin signaling and downstream gene expression.

The in vivo results presented in the current study also indicated that silence of TUG1 could inhibit the CRC tumor growth in the subcutaneously transplanted tumor model, and lower nuclear $\beta$-catenin expression was detected in the excised tumors generated by TUG1 shRNA cells. Furthermore, $\beta$-catenin was also found to be overexpressed in the nuclei of tumor tissues from patients with advanced (stage III and IV) CRC compared with that of stage I and II CRC patients. The results of the present study corresponded with previous studies into CRC $(29,30)$. The aforementioned data indicated that TUG1 serves a notable in an in vivo mouse model, in vitro and in advanced CRC patients.

In conclusion, the results of the present study revealed that TUG1 altered the nuclear localization of $\beta$-catenin, resulting in lowered $\mathrm{Wnt} / \beta$-catenin signaling and the eventual inhibition of proliferation. These results indicated that prevention and treatment of CRC could be performed by targeting the TUG1 and Wnt/ $\beta$-Catenin signaling pathway.

\section{Acknowledgements}

Not applicable.

\section{Funding}

No funding was received.

\section{Availability of data and materials}

The datasets used and/or analyzed during the current study are available from the corresponding author on reasonable request.

\section{Authors' contributions}

JFY was responsible for the study design and the acquisition of data, CYG performed data analysis, $\mathrm{CHX}$ and $\mathrm{HZY}$ performed the functional experiments. ZMW and HYC assisted in designing the experiments and interpret the data. WBL and JFY performed project design and manuscript revisions.

\section{Ethics approval and consent to participate}

The protocols for tumor sample collection were approved by the Institutional Ethics Committee at Nantong Hospital of Traditional Chinese Medicine. Each patient provided written informed consent for inclusion in the present study.

\section{Patient consent for publication}

All the patients provided consent for publication.

\section{Competing interests}

The authors declare that they have no competing interests.

\section{References}

1. Siegel RL, Miller KD and Jemal A: Cancer statistics, 2017. CA Cancer J Clin 67: 7-30, 2017.

2. Watson AJ and Collins PD: Colon cancer: A civilization disorder. Dig Dis 29: 222-228, 2011.

3. Wilusz JE: Long noncoding RNAs: Re-writing dogmas of RNA processing and stability. Biochim Biophys Acta 1859: 128-138, 2015.

4. Sun M, Nie F, Wang Y, Zhang Z, Hou J, He D, Xie M, Xu L, De W, Wang $Z$ and Wang J: LncRNA HOXA11-AS Promotes Proliferation and Invasion of Gastric Cancer by Scaffolding the Chromatin Modification Factors PRC2, LSD1, and DNMT1. Cancer Res 76: 6299-6310, 2016.

5. Okugawa Y, Toiyama Y, Hur K, Toden S, Saigusa S, Tanaka K, Inoue Y, Mohri Y, Kusunoki M, Boland CR and Goel A: Metastasis-associated long non-coding RNA drives gastric cancer development and promotes peritoneal metastasis. Carcinogenesis 35: 2731-2739, 2014.

6. Xia $\mathrm{H}$ and Hui KM: Mechanism of cancer drug resistance and the involvement of noncoding RNAs. Curr Med Chem 21: 3029-3041, 2014

7. Luo HL, Huang MD, Guo JN, Fan RH, Xia XT, He JD and Chen XF: AFAP1-AS1 is upregulated and promotes esophageal squamous cell carcinoma cell proliferation and inhibits cell apoptosis. Cancer Med 5: 2879-2885, 2016.

8. Xue M, Chen W and Li X: Urothelial cancer associated 1: A long noncoding RNA with a crucial role in cancer. J Cancer Res Clin Oncol 142: 1407-1419, 2015.

9. Young TL, Matsuda T and Cepko CL: The noncoding RNA taurine upregulated gene 1 is required for differentiation of the murine retina. Curr Biol 15: 501-512, 2005.

10. Wang XS, Zhang Z, Wang HC, Cai JL, Xu QW, Li MQ, Chen YC, Qian XP, Lu TJ, Yu LZ, et al: Rapid identification of TUG1 as a very sensitive and specific unique marker for human bladder carcinoma. Clin Cancer Res 12: 4851-4858, 2006.

11. Fan Y, Shen B, Tan M, Mu X, Qin Y, Zhang F and Liu Y: Long non-coding RNA TUG1 increases chemoresistance of bladder cancer cells by regulating Wnt signaling. FEBS J 281: 1750-1758, 2014.

12. Tan J, Qiu K, Li M and Liang Y: Double-negative feedback loop between long non-coding RNA TUG1 and miR-145 promotes epithelial to mesenchymal transition and radioresistance in human bladder cancer cells. FEBS Lett 589: 3175-3181, 2015.

13. Tuo YL, Li XM and Luo J: Long noncoding RNA TUG1 modulates colorectal cancer cell growth and apoptosis through decreasing tumor suppressive miR-143. Eur Rev Med Pharmacol Sci 19: 3403-3411, 2015.

14. Huang J, Zhou N, Watabe K, Lu Z, Wu F, Xu M and Mo YY: Long non-coding RNA TUG1 promotes colorectal tumor growth by suppression of p27 (Kip1). Cell Death Dis 5: e1008, 2014. 
15. Zhang EB, Yin DD, Sun M, Kong R, Liu XH, You LH, Han L, Xia R, Wang KM, Yang JS, et al: P53-regulated long non-coding RNA TUG1 affects cell proliferation in human non-small cell lung cancer, partly through epigenetically regulating HOXB7 expression. Cell Death Dis 5: e1243, 2014.

16. Zhang Q, Geng PL, Yin P, Wang XL, Jia JP and Yao J: Down-regulation of long non-coding RNA TUG1 inhibits osteosarcoma cell proliferation and promotes apoptosis. Asian Pac J Cancer Prev 14: 2311-2315, 2013.

17. Isin M, Ozgur E, Cetin G, Erten N, Aktan M, Gezer U and Dalay N: Investigation of circulating lncRNAs in B-cell neoplasms. Clin Chim Acta 431: 255-259, 2014.

18. Xu Y, Wang J, Qiu M, Xu L, Li M, Jiang F, Yin R and Xu L: Upregulation of the long noncoding RNA TUG1 promotes proliferation and migration of esophageal squamous cell carcinoma. Tumour Biol 36: 1643-1651, 2015.

19. Livak KJ and Schmittgen TD: Analysis of relative gene expression data using real-time quantitative PCR and the 2(-Delta Delta C(T)) method. Methods 25: 402-408, 2001.

20. National Research Council (US) Committee for the Update of the Guide for the Care and Use of Laboratory Animals. Guide for the Care and Use of Laboratory Animals. 8th edition. Washington, DC: National Academies Press (US), 2011.

21. Ji Q, Liu X, Fu X, Zhang L, Sui H, Zhou L, Sun J, Cai J, Qin J, Ren J and Li Q: Resveratrol Inhibits invasion and metastasis of colorectal cancer cells via MALAT1 mediated Wnt/ $\beta$-catenin signal pathway. PLoS One 8: e78700, 2013.

22. Venook AP: Advances in Adjuvant Therapy for Colon Cancer: P value or Practical Value. J Clin Oncol 36: 1462-1462, 2018.
23. Gezer U, Ozgur E, Cetinkaya M, Isin M and Dalay N: Long noncoding RNAs with low expression levels in cells are enriched in secreted exosomes. Cell Biol Int 38: 1076-1079, 2014

24. Li ZR, Wu YF, Ma CY, Nie SD, Mao XH and Shi YZ: Down-regulation of c-Myc expression inhibits the invasion of bile duct carcinoma cells. Cell Biol Int 35: 799-802, 2011.

25. Zhang J, Gill AJ, Issacs JD, Atmore B, Johns A, Delbridge LW, Lai $\mathrm{R}$ and McMullen TP: The Wnt/ $\beta$-catenin pathway drives increased cyclin D1 levels in lymph node metastasis in papillary thyroid cancer. Hum Pathol 43: 1044-1050, 2012.

26. Villar J, Cabrera NE, Valladares F, Casula M, Flores C, Blanch L, Quilez ME, Santana-Rodríguez N, Kacmarek RM and Slutsky AS: Activation of the Wnt/ $\beta$-Catenin signaling pathway by mechanical ventilation is associated with ventilator-induced pulmonary fibrosis in healthy lungs. PLoS One 6: e23914, 2011.

27. Zeilstra J, Joosten SP, Dokter M, Verwiel E, Spaargaren M and Pals ST: Deletion of the WNT target and cancer stem cell marker $\mathrm{CD} 44$ in $\mathrm{Apc}(\mathrm{Min} /+)$ mice attenuates intestinal tumorigenesis. Cancer Res 68: 3655-3661, 2008.

28. Brantjes H, Barker N, van Es J and Clevers H: TCF: Lady Justice casting the final verdict on the outcome of Wnt signalling. Biol Chem 383: 255-261, 2002.

29. BrabletzT,Jung A,Reu S,PorznerM,HlubekF,Kunz-SchughartLA, Knuechel R and Kirchner T: Variable betacatenin expression in colorectal cancers indicates tumor progression driven by the tumor environment. Proc Natl Acad Sci USA 98: 10356-10361, 2001.

30. Hlubek F, Brabletz T, Budczies J, Pfeiffer S, Jung A and Kirchner T: Heterogeneous expression of Wnt/beta-catenin target genes within colorectal cancer. Int J Cancer 121: 1941-1948, 2007. 